Open Access

\title{
Surgical site infection in critically ill patients with secondary and tertiary peritonitis: epidemiology, microbiology and influence in outcomes
}

Josep Ballus ${ }^{1}$, Juan C. Lopez-Delgado ${ }^{1 *}$, Joan Sabater-Riera ${ }^{1}$, Xose L. Perez-Fernandez ${ }^{1}$, A. J. Betbese ${ }^{2,3}$ and J. A. Roncal ${ }^{2,3}$

\begin{abstract}
Background: Surgical site infection (SSI) remains a significant problem in the postoperative period that can negatively affect clinical outcomes. Microbiology findings are typically similar to other nosocomial infections, with differences dependent on microbiology selection due to antibiotic pressure or the resident flora. However, this is poorly understood in the critical care setting. We therefore aimed to assess the incidence, epidemiology and microbiology of SSI and its association with outcomes in patients with severe peritonitis in the intensive care unit (ICU).

Methods: We prospectively studied 305 consecutive patients admitted to our surgical ICU from 2010 to 2014 with a diagnosis of secondary or tertiary peritonitis. We collected the following data: SSI diagnosis, demographics, Acute Physiology and Chronic Health Evaluation (APACHE) II score, Simplified Acute Physiology Score (SAPS) II score, type of surgery, microbiology, antibiotic treatment and outcomes. Microbiological sampling was done by means of swabs.

Results: We identified 269 episodes of SSI in 162 patients (53.1\%) aged $64.4 \pm 14.3$ years, of which 200 episodes occurred in men (64.6\%). The mean APACHE II and SAPS II scores were $19.7 \pm 7.8$ and $36.5 \pm 16.1$ respectively. The mean ICU and hospital stays were $19.8 \pm 24.8$ and $21.7 \pm 30$ days respectively. Pseudomonas spp. $(n=52,19.3 \%)$, Escherichia coli $(n=55,20.4 \%)$ and Candida spp. $(n=46,17.1 \%)$ were the most frequently isolated microorganisms, but gram-positive cocci $(n=80,29.7 \%)$ were also frequent. Microorganisms isolated from SSIs were associated with a higher incidence of antibiotic resistance (64.9\%) in ICU patients, but not with higher in-hospital mortality. However, patients who suffered from SSI had longer ICU admissions (odds ratio $=1.024,95 \%$ confidence interval 1.010-1.039, $P=0.001$ ).
\end{abstract}

Conclusions: The incidence of SSI in secondary or tertiary peritonitis requiring ICU admission is very high. Physicians may consider antibiotic-resistant pathogens, gram-positive cocci and fungi when choosing empiric antibiotic treatment for SSI, although more studies are needed to confirm our results due to the inherent limitations of the microbiological sampling with swabs performed in our research. The presence of SSI may be associated with prolonged ICU stays, but without any influence on overall mortality.

Keywords: Surgical site infection, Peritonitis, Intensive care, Outcomes

\footnotetext{
* Correspondence: juancarloslopezde@hotmail.com

'Intensive Care Department, Hospital Universitari de Bellvitge, IDIBELL

(Institut d'Investigació Biomèdica Bellvitge; Biomedical Investigation Institute

of Bellvitge), C/Feixa Llarga s/n, 08907 L'Hospitalet de Llobregat, Barcelona,

Spain

Full list of author information is available at the end of the article
} 


\section{Background}

The skin is the main barrier against bacterial infection of internal tissues, and surgical wounds create a physical disruption to that barrier. The movement of bacteria across the skin barrier can lead to surgical site infections (SSI), one of the most frequent infectious complications of surgical procedures, with the potential risk for adverse outcomes [1]. SSI involves different inflammatory responses that range from low to high clinical significance [2], with that following abdominal surgery being a typical example associated with increased morbidity and mortality [2-5]. Worse still, SSI can spread to surrounding areas and vital deep structures, often requiring debridement or drainage $[3,4]$. Consequently, the treatment of SSI leads to increased costs, especially when we consider the high number of surgical procedures and their complexity in a typical referral hospital [2-5].

Peritonitis, which is defined as inflammation of the serous membrane that covers the abdominal cavity and their organs, is classified into primary (spontaneous), secondary (process-related pathology in the visceral organs) and tertiary (persistent or recurrent after initial adequate surgical treatment). Secondary and tertiary peritonitis are associated with higher morbidity with mortality rates of $17-63 \%[6,7]$. Tertiary peritonitis usually occurs in ICU settings at least 48-72 h following adequate treatment of secondary peritonitis, and has a mortality rate of $30-60 \%$ [8].

Centers for Disease Control and Prevention (CDC) estimates that the risk of SSI associated with abdominal surgery ranges from approximately 2 to $8 \%$, depending on the type of surgery $[2,3,9,10]$. SSI is classified into several categories: clean (2\%), clean-contaminated (3\%), contaminated (6\%) and dirty (7\%) [2, 11]. Stratification before surgery could help identify at-risk patients suitable for surveillance [3].

Despite the marked influence of SSI associated with severe peritonitis on public health and clinical practice, it has been poorly addressed in the literature, even in the ICU setting. This study therefore aimed to describe the incidence, epidemiology, microbiology and outcomes of SSI in patients admitted with secondary or tertiary peritonitis to the ICU of a tertiary referral hospital.

\section{Materials and methods}

This prospective, observational study was carried out at from January 2010 to December 2014. At the time this study was performed, the Hospital Universitari de Bellvitge (HUB) was a tertiary hospital with 850 general care beds and 44 ICU beds. We included all consecutive patients from any type of abdominal surgery who required ICU admission beyond $72 \mathrm{~h}$ for secondary or tertiary peritonitis. All patients received standard preoperative hygiene care and antibiotic prophylaxis at anaesthetic induction consistent with our institutional protocols for elective and emergency surgery [4].

SSI was defined using the CDC definition [11] and diagnosis was by the responsible physicians, based on clinical criteria. Any purulent drainage from a surgical incision with signs of inflammation of the surrounding tissue was considered an SSI, whether microorganisms were isolated in cultures or not. The infection had to present at the surgical site within 30 days of surgery [2-4]. The study was approved by the Institutional Ethics Committee of our hospital (Comité d' Ètica i Assajos Clínics de HUB (CEIC); Ethics and Clinical Assays Committee of HUB), and informed consent was waived due to the observational design.

In all patients, the decisions regarding ICU admission and treatment were made by the treating physician. Data were recorded from the medical registry of each patient in real time, using a standardised questionnaire, and collected in a database. The following information was recorded on admission: demographic data, medical history and comorbidities, surgical indication and type of surgery (elective or emergency), surgical technique, intraoperative variables (number of drains inserted), microbiologic findings, arterial lactate on admission and treatment characteristics. During their ICU stay, we also recorded the following: need for vasopressor drugs, mechanical ventilation and renal replacement therapy (RRT) and; any new microbiological findings, including the appropriateness, changes and resistance to antibiotic treatment. Illness severity was quantified with the Acute Physiology and Chronic Health Evaluation (APACHE) and Simplified Acute Physiology Score (SAPS) scoring systems during the first $24 \mathrm{~h}$ of ICU admission for all patients. After ICU discharge, follow-up was planned to collected data on in-hospital mortality and patients were followed until discharge from ICU or until resolution.

The surgical teams collaborated with ICU physicians to control the SSI, using simple washouts or serial debridements when appropriate. We obtained tissue samples and exudate samples, and direct needle aspiration was used when needed, in collaboration with the surgical team. The microbiological samples were obtained under conditions as sterile as possible in order to avoid colonizers of the superficial wound. The deepness of tissue sample was evaluated based on SSI characteristics. If necessary, drainage was performed, and any necrotic tissue was debrided and foreign material removed. Intensive irrigation with saline solution was employed when necessary to facilitate mechanical debridement [2]. We provided rational antibiotic therapy based on local guidelines and after consultation with an infectious disease physician.

For diagnosis purposes, microbiological samples were sent to the laboratory as swabs in culture media for 
semiquantitative aerobic and anaerobic cultures. To isolate anaerobes, specimens were inoculated into Columbia blood agar plates enriched with hemin and menadione, incubated in an anaerobic chamber at $37{ }^{\circ} \mathrm{C}$, and specimens were Gram stained at 48 and $96 \mathrm{~h}$ for direct examination.

Statistical analysis was conducted using PASW Statistics 13.0 (SPSS Inc., Chicago, Illinois, USA). Continuous data are expressed as mean \pm standard deviation and categorical data are expressed as percentages. Comparisons between groups with non-normal distributions were by two-sample $t$-tests or Mann-Whitney $U$ tests after applying the one-sample Kolmogorov-Smirnov test. The $X^{2}$-test was used to evaluate categorical variables. Multivariable analysis was done to assess the influence of SSI and other SSI-related factors, such as the microbiology results, on mortality and outcomes. Odds ratios (ORs) and $95 \%$ confidence intervals (CIs) are quoted as appropriate. A $P$-value of 0.05 was considered statistically significant in all cases.

\section{Results}

Of the 305 patients hospitalised for secondary or tertiary peritonitis in our ICU, we identified 269 episodes of SSI in 162 patients. The SSI rate of $53.1 \%$ was higher in ICU compared with the rest of hospitalized patients who underwent major abdominal surgery $(n=193 / 935$; $20.6 \%)$ during the study period $(P<0.001)$. Patient characteristics, inflammatory response, type of surgery and outcomes are shown in Table 1. The mean duration of hospitalisation prior to surgery was $8.9 \pm 2.5$ days. Urgent abdominal surgery comprised $28 \%-35 \%$ of all abdominal surgeries performed at our hospital, showing a difference in the type of surgery in comparison with ICU patients $(P=0.01)$. The types of surgery (based on the anatomical location) of the different identified SSI episodes are shown in Fig. 1. All patients were monitored with a central venous catheter, arterial catheter and urinary catheter, and all patients were on vasoactive drugs or inotropic support.

In the studied population, we identified 1181 positive culture isolates: $269(22.7 \%)$ were from SSIs, 486 $(41.2 \%)$ were from intra-abdominal abscesses, 251 (21.2\%) were from positive blood cultures (blood cultures) and $175(14.9 \%)$ were from other cultures. The microbiology results for isolates from SSI are shown in Table 2, with a preponderance of Escherichia coli $(n=55,20.4 \%)$ and Pseudomonas aeruginosa $(n=52$, $19.3 \%$ ), while gram-positive cocci and Candida spp. were also frequent at rates of $29.7 \%(n=80)$ and $17.1 \%(n=46)$ respectively. Antibiotic resistance to two or more antibiotics occurred in $64.9 \%(n=174)$, with rates of Extended spectrum beta-lactamase-producing Enterobacteriaceae $(n=30)$ and Pseudomonas aeruginosa carbapenem-resistant $(n=32)$ of $11.1 \%$ and $11.9 \%$ respectively, but with low rates of typical multi-resistant microorganisms such as Acinetobacter baumanii (2.9\%, $n=8)$ and methicillin resistant Staphylococcus aureus (MRSA) $(2.2 \%, n=6)$. The microorganisms isolated from intra-abdominal abscesses were the same of those isolated in SSI samples in the $20.9 \%$ of the patients $(n=34)$ with similar rates of of multi-resistant bacteria. No relationship was established between the intraabdominal abscesses and the occurrence of SSI based of clinical and surgical evaluation. Based on the culture antibiograms, $99.5 \%$ of patients received appropriate antibiotic treatment. The most commonly used antibiotics are shown in Table 2, with the use of multiple, simultaneous or sequential antibiotics being used in $72.5 \%$ of the cases $(n=195)$. A mean of 2.6 antibiotics was used per SSI, with treatment lasting $14 \pm 8$ days.

Total mortality was $37.9 \%(n=116)$, from which $27.9 \%(n=85)$ correspond to ICU deaths. Although SSI was not associated with higher mortality in our population when confounders, such as variables that reflected disease severity, were included in the multivariate analysis, it was associated with a longer ICU stay $(\mathrm{OR}=1.024,95 \% \mathrm{CI}$ : $1.010-1.039 ; P=0.001$ ). Indeed, mortality was lower in the group with SSI $(\mathrm{OR}=0.547,95 \%$ CI 0.323 $0.924 ; P=0.024)$. The need for RRT $(\mathrm{OR}=3.358$, $95 \%$ CI: 1.619-6.966; $P=0.001)$ and prolonged ICU stay $(\mathrm{OR}=1.071,95 \%$ CI: $1.046-1.097 ; P<0.001)$ were associated with higher in-hospital mortality.

\section{Discussion}

This study provides data on the incidence and microbiology of SSIs for a large cohort of critically ill patients admitted with secondary or tertiary peritonitis to a surgical ICU. It confirms that there was a high incidence of SSI in those patients. The main findings of our study were that SSI was associated with a prolonged ICU stay, but that it had little impact on the overall in-hospital mortality in our population.

The development of postoperative SSI is known to have been multifactorial, arising from a complex relationship between host and environmental factors [1, 2]. Host risk factors for SSI include morbid obesity, disease severity, advanced age, low blood-protein levels and malnutrition, diabetes, malignancy and sepsis, while other risk factors that include susceptibility include immunosuppression, smoking and having a distant infection site [10]. Pre-existing morbidity, the time of surgery and the type of SSI may also play key roles in the development of SSIs [10]. Thus, an increasingly elderly population with a greater number of comorbidities significantly increases the risk of developing an SSI [9, 12]. Critically ill patients represent an increasing proportion of the inpatient population that will undoubtedly lead to greater 
Table 1 Patient characteristics, inflammatory response, type of surgery and outcomes

\begin{tabular}{|c|c|c|c|c|}
\hline & All patients $(n=305)$ & Non-SSI subgroup ( $n=143 ; 46.9 \%)$ & SSI subgroup ( $n=162 ; 53.1 \%)$ & $P$ \\
\hline Age (years) & $64.4 \pm 14.3$ & $63.5 \pm 14.4$ & $65.3 \pm 13.8$ & 0.27 \\
\hline Sex (male/ female) & $200(65.6 \%) / 105(34.4 \%)$ & $86(60.1 \%) / 57$ (39.9\%) & $114(70.4 \%) / 48$ (29.6 \%) & 0.07 \\
\hline APACHE II & $19.7 \pm 7.8$ & $20.5 \pm 8.7$ & $18.95 \pm 6.6$ & 0.07 \\
\hline SAPS ॥ & $36.5 \pm 17.1$ & $37.9 \pm 19.3$ & $35.1 \pm 13.9$ & 0.13 \\
\hline Immunosuppression & $36(11.8 \%)$ & $18(12.6 \%)$ & $18(11.1 \%)$ & 0.72 \\
\hline Neutropenia on admission & $14(4.6 \%)$ & 7 (4.9\%) & 7 (4.3\%) & 1.00 \\
\hline HIV & $37(12.1 \%)$ & 19 (13.3\%) & $18(11.1 \%)$ & 0.60 \\
\hline \multicolumn{5}{|l|}{ Type of infection } \\
\hline Secondary peritonitis & $140(45.9 \%)$ & $71(49.6 \%)$ & $69(42.6 \%)$ & 0.09 \\
\hline Tertiary peritonitis & $165(54.1 \%)$ & $72(50.4 \%)$ & $93(57.4 \%)$ & 0.023 \\
\hline \multicolumn{5}{|l|}{ Inflammatory response on admission } \\
\hline Sepsis & $27(8.8 \%)$ & $11(7.7 \%)$ & $16(9.8 \%)$ & 0.85 \\
\hline Severe sepsis & $32(10.5 \%)$ & $15(10.5 \%)$ & $17(10.5 \%)$ & 1.00 \\
\hline Septic shock & $204(67 \%)$ & 89 (62.2 \%) & 115 (71.1\%) & 0.006 \\
\hline Multi-organ failure & $42(13.7 \%)$ & $28(19.5 \%)$ & $14(8.6 \%)$ & 0.003 \\
\hline \multicolumn{5}{|l|}{ Surgery data } \\
\hline Urgent surgery & $276(90.5 \%)$ & $125(87.4 \%)$ & $151(93.2 \%)$ & 0.11 \\
\hline Percutaneous surgery & $41(13.5 \%)$ & $17(11.8 \%)$ & $24(14.8 \%)$ & 0.10 \\
\hline Laparoscopic surgery & $10(3.3 \%)$ & $4(2.8 \%)$ & $6(3.7 \%)$ & 0.28 \\
\hline Laparotomy surgery & $231(75.7 \%)$ & $112(78.5 \%)$ & $119(73.5 \%)$ & 0.12 \\
\hline Combined surgery & $23(7.5 \%)$ & $10(6.9 \%)$ & $13(8 \%)$ & 0.14 \\
\hline Number of drainages on ICU admission & $2 \pm 1.7$ & $1.2 \pm 1.7$ & $2.3 \pm 1.8$ & 0.01 \\
\hline \multicolumn{5}{|l|}{ Outcomes } \\
\hline ARDS & $121(39.6 \%)$ & $55(38.4 \%)$ & $66(40.7 \%)$ & 0.85 \\
\hline On mechanical ventilation & $279(91.5 \%)$ & $130(90.9 \%)$ & 149 (92\%) & 0.83 \\
\hline Arterial lactate on admission $\left(\mathrm{mmol} \cdot \mathrm{dL}^{-1}\right)$ & $4.3 \pm 3.3$ & $4.1 \pm 3.9$ & $5.3 \pm 2.5$ & 0.57 \\
\hline RRT & $77(25.2 \%)$ & $40(28 \%)$ & $37(22.8 \%)$ & 0.35 \\
\hline Total parenteral Nutrition needs (any time) & $251(82.3 \%)$ & $113(79 \%)$ & $138(85.2 \%)$ & 0.17 \\
\hline ICU stay (days) & $19.8 \pm 24.8$ & $12.9 \pm 14.2$ & $22.3 \pm 32.9$ & 0.002 \\
\hline Hospital stay (days) & $21.7 \pm 30$ & $17.6 \pm 35.3$ & $25.4 \pm 26.5$ & 0.031 \\
\hline In-hospital mortality & $115(38 \%)$ & $64(45.1 \%)$ & 110 (31.7\%) & 0.018 \\
\hline
\end{tabular}

Data are mean \pm standard deviation or percentage

SAPS simplified acute physiology score, APACHE acute physiology and chronic health evaluation, HIV human immunodeficiency virus, ARDS acute respiratory distress syndrome, RRT renal replacement therapy, ICU intensive care unit

diagnostic and management challenges, especially given that most SSIs in the ICUs are nosocomial [9]. SSIs are most common in high-risk patients, with an incidence of about $11.7 \%$ [12, 13].

Antibiotic prophylaxis reduces postoperative morbidity and length of hospital stay, which positively affects SSIrelated costs [4]. Wounds with a risk of infection below $2 \%$ do not generally require antibiotic prophylaxis, but notable exceptions include the placement of a prosthesis, cardiovascular surgery and neuro-surgery [14]. Up to $15 \%$ of all elective surgical patients may develop an SSI, with rates as high as $30 \%$ being common in contaminated or dirty surgical procedures [5]. In our study, the majority of procedures were considered dirty or contaminated, and many of the critically ill patients had markedly decreased serum protein concentrations. Together, these may ultimately explain our higher SSI rate. Our higher rates may also reflect the inherent risks of tertiary care institutions and the severity of our cohort. Therefore, our results may not be applicable to secondary and non-referral hospitals.

An SSI can increase hospital stay by about six days and can add $10-20 \%$ to hospital costs, even leading to death; therefore, prevention and control should be an 


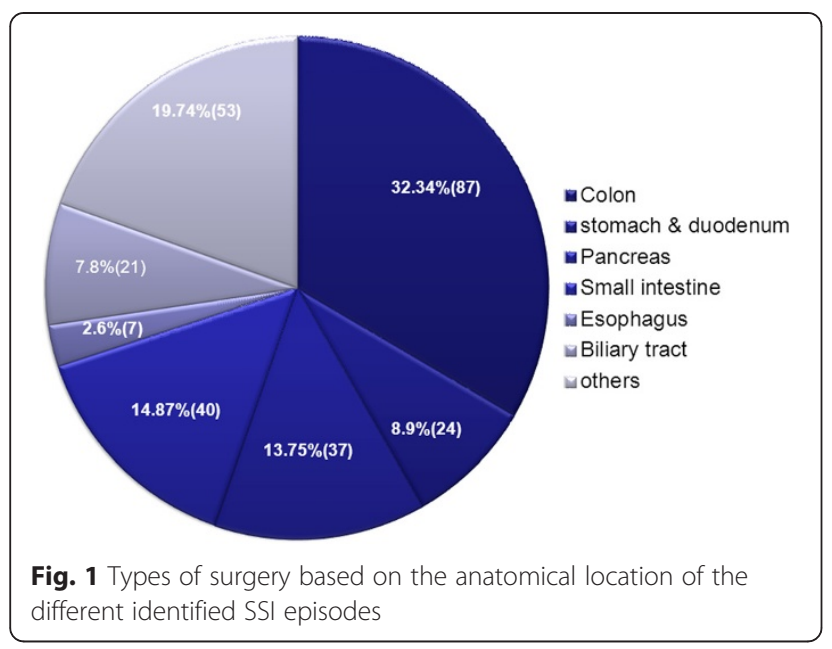

Table 2 Microbiology cultures and antibiotic therapy of surgical site infection

\begin{tabular}{ll}
\hline Isolated microorganisms & \\
\hline Escherichia coli & $20.4 \%(n=55)$ \\
Pseudomonas aeruginosa & $19.3 \%(n=52)$ \\
Candida albicans & $13.7 \%(n=37)$ \\
Staphylococcus coagulase negative & $12 \%(n=32)$ \\
Staphylococcus epidermidis & $7.4 \%(n=20)$ \\
Enterococcus faecalis & $4.4 \%(n=12)$ \\
Candida spp. (Other than C. albicans) & $3.3 \%(n=9)$ \\
Acinetobacter baumanii & $2.9 \%(n=8)$ \\
Klebsiella spp. & $2.6 \%(n=7)$ \\
Proteus mirabilis & \\
Methicillin resistant Staphylococcus aureus & \\
Staphylococcus aureus & $2.2 \%(n=6)$ \\
Enterococcus faecium & $1.8 \%(n=5)$ \\
Enterobacter cloacae & \\
Clostridium spp. & $1.6 \%(n=4)$ \\
Enterobacter aerogenes & \\
Other & \\
Antibiotic therapy & $1.1 \%(n=3)$ \\
Meropenem & \\
Piperacillin-tazobactam & $7.7 \%(n=54)$ \\
Teicoplanin & $6.7 \%(n=48)$ \\
Fluconaillin & $4.8 \%(n=34)$ \\
Ertapenem & $6 \%(n=42)$ \\
\hline Othipener &
\end{tabular}

important component of healthcare quality control $[3,4]$. SSIs may occur following any surgical incision, even after the use of minimally invasive techniques, so SSIs need to be reported through systematic monitoring programmes for nosocomial infection [15]. We showed that patients suffering from SSI in our cohort had longer ICU stays. However, we do not think this was simply a surrogate of higher illness severity in the SSI group because of the comparable severity scores between groups.

The dominant causative microorganisms and treatment options have changed over time. Today, most common pathogens are resistant to common antibiotics [16] with the need for a high index of suspicion, prompt operative intervention, appropriate antibiotic treatment and proper resuscitation [5]. Hypovolemia and hypothermia create peripheral vasoconstriction that leads to poor tissue perfusion, which facilitates the development of SSI in the presence of necrotic tissue, foreign bodies, hematomas and seromas [2]. The microbiology of intra-abdominal infections also varies depending on the source of infection, prior use of antibiotics, the site of infection and if it is community acquired or nosocomial.

Besides the host and wound factors, physicians should be aware of the increase in high-virulence species, such as Staphylococcus aureus and Streptococcus pyogenes. In addition, Escherichia coli, Bacteroides fragilis and other gram-negative, anaerobic pathogens are common in large bowel perforations [16]. Nosocomial intra-abdominal infections often involve microorganisms such as Pseudomonas spp., Enterococcus spp. and fungi [5]. In our population, there was an increased presence of multidrugresistant pathogens and fungal SSI rates when compared with other series $[17,18]$. This could be explained by the higher antibiotic pressure, use of broad-spectrum antibiotics, illness severity and prolonged treatment periods. Indeed, concomitant treatment for peritonitis compounded matters. We also showed higher reliance on total parenteral nutrition (TPN) because enteral nutrition was poorly tolerated and we opted to initiate it early to avoid hypoalbuminemia, which is a risk factor for fungal infection and SSI in critically ill patients [19]. However, we concede that TPN is a risk factor for all types of fungal infection in ICU, especially among surgical patients [20].

The increasing trend to reduce hospital stays by implementing innovative surgical techniques (particularly minimally invasive and endoscopic procedures) makes it necessary to ensure that accurate measurement and monitoring of adverse events can take place after discharge. Without doing so, we cannot establish the real impact of SSI on morbidity and mortality $[3,9,12]$. Control measures with an emphasis on the education of healthcare professionals, such as frequent hand washing and the need to isolate patients with multi-resistant bacteria in cluster units, are necessary to reduce SSI rates 
[21]. Although the total elimination of SSI is not possible, a reduction in the rate of infection to a minimum should be achievable, even in critically ill patients [4].

Our study presents certain limitations. The most important is that we used for microbiological sampling a skin swabs instead of the "gold standard" for culture of skin, which are tissue biopsy or aspiration sampling of infected tissue. We could have obtained colonizer microorganisms that are not responsible for the infection and cultures may be misleading organisms in the deep tissue infection. Thus, our results should be considered cautiously and more studies are needed to confirm them. Secondly, this was a single-centre observational study and our results cannot be extrapolated to other less severely ill populations. Among the strengths of this study are the large sample size, the prospective entry of all data and the use of postoperative scores, which are not used in contemporary studies, even though their importance in risk stratification has been emphasised over recent decades. Furthermore, this investigation was conducted at a large tertiary referral hospital with a high level of complexity over a four-year period.

\section{Conclusions}

In summary, the incidence of SSI was very high in patients with secondary or tertiary peritonitis requiring ICU admission. When they prescribe antibiotic therapy, physicians should consider that microorganisms isolated from patients with SSI are more likely to include multidrugresistant pathogens, including Pseudomonas spp., grampositive cocci and fungi, although more studies are needed to confirm our results due to the inherent limitations of the microbiological sampling with skin swabs performed in our research. Despite the presence of an SSI may be associated with prolonged ICU stays, we did not find any effect on the in-hospital mortality in our population.

\section{Abbreviations}

SSI: Surgical site infection; ICU: Intensive care unit; APACHE: Acute physiology and chronic health evaluation; SAPS: Simplified acute physiology score; RRT: Renal replacement therapy; TPN: Total parenteral nutrition; ARDS: Acute respiratory distress syndrome in adults.

\section{Competing interests}

There is no funding support or conflicts of interest for the present paper.

\begin{abstract}
Authors' contributions
$\mathrm{JB}$ was involved in the conception and design of the research, acquisition of data and wrote the paper. JCLD performed partial statistical analysis, acquisition of data and wrote the paper. JSR contributed to the design of the research and acquisition of data. XLPF supervised and performed statistical analysis. AJB was involved in the conception, design of the research and interpretation of data. JAR was involved in the design of the research and supervised the writing of the present manuscript. All authors read and approved the final version of this manuscript.
\end{abstract}

\section{Acknowledgments}

The authors wish to thank the ICU nurses and all members of the general surgery department for their contribution to the study and for their care of the patients reported in this paper.

\section{Author details}

${ }^{1}$ Intensive Care Department, Hospital Universitari de Bellvitge, IDIBELL (Institut d'Investigació Biomèdica Bellvitge; Biomedical Investigation Institute of Bellvitge), C/Feixa Llarga s/n, 08907 L'Hospitalet de Llobregat, Barcelona, Spain. ${ }^{2}$ Intensive Care Department, Hospital de la Santa Creu i Sant Pau, Barcelona, Spain. ${ }^{3}$ Universitat Autònoma de Barcelona, C/Sant Quintín 89, 08041 Barcelona, Spain.

Received: 27 January 2015 Accepted: 22 July 2015

Published online: 30 July 2015

\section{References}

1. Giacomettia A, Cirioni O, Schimizzi AM, Del Prete MS. Epidemiology and microbiology of surgical wound infections. J Clin Microbiol. 2000;38:918-22.

2. Leaper DJ. Risk factors for and epidemiology of surgical site infections. Surg Infect (Larchmt). 2010;11:283-7.

3. Oluwatosin OM. Surgical wound infection: a general overview. Ann Ibadan Postgrad Med. 2005;3:26-30.

4. L'Ecuyer PB, Murphy D, Russell Little J, Fraser VJ. The epidemiology of chest and Leg wound infections following cardiothoracic surgery. Clin Infect Dis. 1996:22:424-9.

5. Barie PS. Surgical site infections: epidemiology and prevention. Surg Infect (Larchmt). 2002;1:S9-21.

6. de Ruiter J, Weel J, Manusama E, Kingma WP, van der Voort PH. The epidemiology of intra-abdominal flora in critically ill patients with secondary and tertiary abdominal sepsis. Infection. 2009:37:522-7.

7. Marshall JC, Innes M. Intensive care unit management of intra-abdominal infection. Crit Care Med. 2003;31:2228-37.

8. Anaya DA, Nathens AB. Risk factors for severe sepsis in secondary peritonitis. Surg Infect (Larchmt). 2003;4:355-62.

9. Stafford RE, Weigelt JA. Surgical infections in the critically ill. Curr Opin Crit Care. 2002;8:449-52.

10. Nakamura T, Mitomi H, Ihara A, Onozato W, Sato T, Ozawa H, et al. Risk factors for wound infection after surgery for colorectal cancer. World J Surg. 2008;32:1138-41.

11. Horan TC, Gaynes RP, Martone WJ, Jarvis WR, Emori TG. CDC definitions of nosocomial surgical site infections, 1992: a modification of CDC definitions of surgical wound infections. Infect Control Hosp Epidemiol. 1992;1992(13):606-8.

12. Rubin RH. Surgical wound infection: epidemiology, pathogenesis, diagnosis and Management. BMC Infect Dis. 2006;6:171-2.

13. Knaus WA, Draper EA, Wagner DP, Zimmerman JE. APACHE II: a severity of disease classification system. Crit Care Med. 1985;13:818-29.

14. Sawyer RG, Raymond DP, Pelletier SJ, Crabtree TD, Gleason TG, Pruett TL. Implications of 2,457 consecutive surgical infections entering year 2000. Ann Surg. 2001;233:867-74

15. Coello R, Charlett A, Wilson J, Ward V, Pearson A, Borriello P. Adverse impact of surgical site infections in English hospitals. J Hosp Infect. 2005;60:93-103.

16. Emori TG, Culver DH, Horan TC. National nosocomial infections surveillance system (NNIS): description of surveillance methods. Am J Infect Control. 1991;19:19-35.

17. García-Sánchez JE, García-García MI, García-Garrote F, Sánchez-Romero I. Microbiological diagnosis of intra-abdominal infections. Enferm Infecc Microbiol Clin. 2013;31:230-9.

18. Janda JM, Abbott SL, Brenden RA. Overview of the etiology of wound infections with particular emphasis on community-acquired illnesses. Eur J Clin Microbiol Infect Dis. 1997;16:189-201.

19. Yang SP, Chen YY, Hsu HS, Wang FD, Chen LY, Fung CP. A risk factor analysis of healthcare-associated fungal infections in an intensive care unit: a retrospective cohort study. BMC Infect Dis. 2013;13:10.

20. Morace $\mathrm{G}$, Borghi E. Fungal infections in ICU patients: epidemiology and the role of diagnostics. Minerva Anestesiol. 2010;76:950-6.

21. Olson M, Lee JT. Continuous, 10-year wound infection surveillance: results, advantages, and unanswered questions. Arch Surg. 1990;125:794-803. 